\title{
Optimizing the Joint Transmit and Receive MMSE Design Using Mode Selection
}

\author{
Nadia Khaled, Student Member, IEEE, Steven Thoen, and Luc Deneire, Member, IEEE
}

\begin{abstract}
To approach the potential multiple-input multiple-output (MIMO) capacity while optimizing the system bit-error rate (BER) performance, the joint transmit and receive minimum mean squared error (joint $\mathbf{T} \mathbf{x} / \mathbf{R x}$ MMSE) design has been proposed. It is the optimal linear scheme for spatial multiplexing MIMO systems, assuming a fixed number of spatial streams $p$ as well as fixed modulation and coding across these spatial streams. However, the number of spatial streams has been arbitrarily chosen and fixed, which may lead to an inefficient power allocation strategy and a poor BER performance. In this paper, we relax the constraint of fixed number of streams $p$ and optimize this value for the current channel realization, under the constraints of fixed average total transmit power $P_{T}$ and fixed rate $R$, what we refer to as mode selection. Based on the observation of the existence of a dominant optimal number of streams value for the considered Rayleigh flat-fading MIMO channel model, we further propose an "average" mode selection that avoids the per-channel adaptation through using the latter dominant value for all channel realizations. Finally, we exhibit the significant BER improvement provided by our mode selection over the conventional joint Tx/Rx MMSE design. Such significant improvement is due to the better exploitation of the MIMO spatial diversity and the more efficient power allocation enabled by our mode selection.
\end{abstract}

Index Terms - Joint transmit and receive optimization, Rayleigh flat-fading MIMO channels, selection, spatial multiplexing.

\section{INTRODUCTION}

$\mathbf{M}$ ULTIPLE-INPUT multiple-output (MIMO) wireless channels have significantly higher capacities than conventional single-input single-output (SISO) channels [1]. These capacities are related to the multiple parallel spatial subchannels that are opened through the use of multiple antennas at both the transmitter and the receiver. Spatial multiplexing (SM) is a technique that transmits multiple independent parallel data streams on these available spatial subchannels in an attempt to approach the MIMO capacity [2], [3].

Two main design trends have emerged that enable spatial multiplexing corresponding to whether channel state information (CSI) is available or not at the transmitter. On the one hand, BLAST-like space-time techniques [3], [4] make use of

Paper approved by A. Lozano, the Editor for Wireless Communication of the IEEE Communications Society. Manuscript received March 1, 2003; revised September 22, 2003, and July 15, 2004. This work was presented in part at the 4th IEEE Workshop on Signal Processing Advances in Wireless Communications (SPAWC'03), Rome, Italy, June, 2003.

N. Khaled is with the IMEC vzw, B-3001 Leuven, Belgium (e-mail: nadia.khaled@imec.be).

S. Thoen is with the RFMD, B-3001 Leuven, Belgium (e-mail: steven.thoen@rfmd.com).

L. Deneire is with the IUT-GTR Universite de Nice, Laboratoire I3S, F-06903 Sophia Antipolis Cedex, France (e-mail: deneire@unice.fr)

Digital Object Identifier 10.1109/TCOMM.2005.844923 the available transmit antennas to transmit as many independent data streams and do not require CSI at the transmitter. On the other hand, the joint transmit and receive space-time processing techniques take advantage of the potentially available CSI at both sides of the link to maximize the system's information rate [9] or alternatively reduce the system's bit-error rate (BER) [5]-[8] under a fixed rate constraint.

In this contribution, we adopt the second approach, namely joint transmit and receive space-time processing, based on the assumption that the channel is slowly varying and hence CSI can be acquired through either feedback or plain channel estimation in TDD-based systems. Furthermore, among the possible design criteria, we retain the joint transmit and receive minimum mean squared error (joint Tx/Rx MMSE) criterion, initially proposed in [5] and further discussed in [7] and [8], for it is the optimal linear solution for fixed coding and modulation across the spatial streams. This constraint is set to reduce the system's complexity and adaptation requirements in comparison to the optimal yet complex bit loading strategy [10].

For the flat-fading $\left(M_{T}, M_{R}\right)$ MIMO system herein considered, assuming a fixed number of spatial multiplexing data streams, $p \leq \operatorname{Min}\left(M_{T}, M_{R}\right)$, and a fixed symbol constellation across these data streams, the conventional joint $\mathrm{Tx} / \mathrm{Rx}$ MMSE design devises an optimal filter-pair $\{\mathbf{T}, \mathbf{R}\}$. The latter filter-pair solution was shown [7], [8] to perform the singular value decomposition (SVD) of the MIMO channel matrix, what boils down to decoupling the MIMO channel into multiple parallel spatial subchannels among which the $p$ strongest are used to convey the available $p$ data streams. The joint Tx/Rx MMSE design also proposes an optimum transmit power allocation policy among the decoupled spatial subchannels. It allocates power only to a selection of subchannels that are above a given signal-to-noise ratio (SNR) threshold imposed by the transmit power constraint. Furthermore, more power is given to the weaker modes of the previous selection. It is clear that the data streams assigned to the nonselected spatial subchannels are lost leading to a high MMSE and consequently a poor BER performance. Moreover, the arbitrary and initial choice of the number of streams $p$ may lead to the use of weak modes that consume most of the power and detrimentally impact the other modes. The previous remarks show the impact of the choice of $p$ on the power allocation efficiency as well as on the BER performance of the joint Tx/Rx MMSE design. Hence, it is relevant to consider the number of streams $p$ as an additional design parameter rather than a mere arbitrary fixed scalar as in [6]-[8].

Consequently, in this paper, we address the issue of optimizing the number of streams $p$ of the joint Tx/Rx MMSE according 

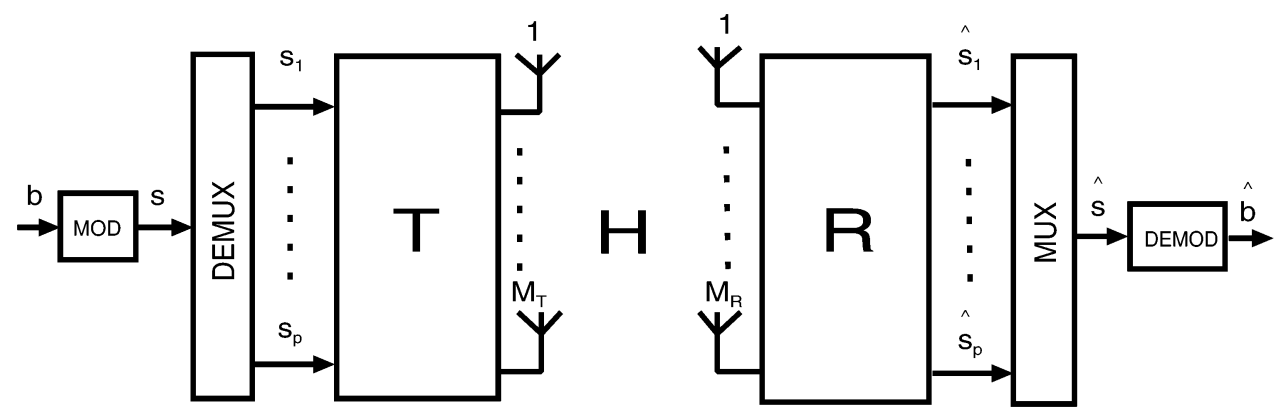

Fig. 1. Considered $\left(M_{T}, M_{R}\right)$ spatial multiplexing MIMO system using joint linear transmit and receive optimization.

to the available CSI, under the constraints of fixed average total transmit power and fixed rate for Rayleigh flat-fading MIMO channels. A remark in [7] also raised this issue without pursuing it.

The rest of the paper is organized as follows. Section II provides the system model and describes the conventional joint Tx/Rx MMSE design of [6]. Based on that, our mode selection approach is introduced in Section III and further analyzed in Section IV. The BER improvements enabled by the proposed mode selection are assessed in Section V. Finally, the conclusions are drawn in Section VI.

\section{System Model AND PRELIMINARIES}

In this section, we first introduce the system model used throughout the paper. Then, we describe the conventional joint $\mathrm{Tx} / \mathrm{Rx}$ MMSE design previously proposed for high data-rate single-user MIMO transmissions [6], which represents the baseline design for our optimization.

\section{A. Notations}

In this paper, normal letters indicate scalar quantities, boldface letters represent vectors and boldface uppercase letters designate matrices. $\mathbf{I}_{p}$ is the $p \times p$ identity matrix. Moreover, $\operatorname{trace}(\mathbf{X})$ and $[\mathbf{X}]_{i, j}$ respectively stand for the trace and $(i, j)$ th entry of matrix $\mathbf{X} .[x]^{+}$refers to $\operatorname{Max}(x, 0)$ and $(\cdot)^{*}$ denotes the conjugate transpose of a vector or a matrix. Finally, $\|\mathbf{x}\|_{2}$ indicates the 2-norm of vector $\mathbf{x}$.

\section{B. System Model}

The single-user spatial-multiplexing MIMO communication system under consideration is depicted in Fig. 1. It represents a transmitter and a receiver, both equipped with multiple-element antennas and assumed to have perfect knowledge about the current channel realization. The transmitter first modulates the transmit bit stream $b$ according to a pre-determined symbol constellation of size $M_{p} \cdot{ }^{1}$ Then, it demultiplexes the output symbols into $p \leq \operatorname{Min}\left(M_{T}, M_{R}\right)$ independent streams. This spatial multiplexing operation actually converts the serial symbol stream $s$ into $p$ parallel symbol streams or equivalently into a higher-dimensional symbol stream where every symbol now is a $p$-dimensional spatial symbol, for instance $\mathbf{s}(k)$ at discrete-time index $k$. These spatial symbols are then

${ }^{1}$ This implies the same symbol constellation across all spatial streams. pre-filtered by the transmit filter $\mathbf{T}$ and sent onto the MIMO channel through the $M_{T}$-element transmit antenna. At the receiver, the $M_{R}$ symbol-sampled complex baseband outputs of the $M_{R}$-element receive antenna are post-filtered by the receive filter $\mathbf{R}$. The $p$ output streams conveying the detected spatial symbols $\hat{\mathbf{s}}(k)$ are then multiplexed and demodulated to recover the initially transmitted bit stream. For a flat-fading MIMO channel, the global system equation is given by

$$
\underbrace{\left[\begin{array}{c}
\hat{s}_{1}(k) \\
\vdots \\
\hat{s}_{p}(k)
\end{array}\right]}_{\hat{\mathbf{s}}(k)}=\mathbf{R H T} \underbrace{\left[\begin{array}{c}
s_{1}(k) \\
\vdots \\
s_{p}(k)
\end{array}\right]}_{\mathbf{s}(k)}+\mathbf{R} \underbrace{\left[\begin{array}{c}
n_{1}(k) \\
\vdots \\
n_{M_{R}}(k)
\end{array}\right]}_{\mathbf{n}(k)}
$$

where $\mathbf{n}(k)$ is the $M_{R}$-dimensional receiver noise vector at discrete-time index $k . \mathbf{H}$ is the $M_{R} \times M_{T}$ channel matrix whose $(i, j)$ th entry, $[\mathbf{H}]_{i, j}$, represents the complex channel gain from the $j$ th transmit antenna element to the $i$ th receive antenna element. In the sequel, the discrete-time index $k$ is dropped for notational brevity.

\section{Conventional Joint Tx/Rx MMSE Design}

The transmit and receive filters, $\mathbf{T}$ and $\mathbf{R}$, represented by an $M_{T} \times p$ and $p \times M_{R}$ matrix, respectively, are jointly designed to minimize the sum mean squared error on the spatial symbols s subject to fixed average total transmit power $P_{T}$ constraint as stated in [6]

$$
\begin{cases}\operatorname{Min}_{\mathbf{R}, \mathbf{T}} & E_{\mathbf{s}, \mathbf{n}}\left\{\|\mathbf{s}-(\mathbf{R H T} \mathbf{s}+\mathbf{R n})\|_{2}^{2}\right\} \\ \text { subject to }: & E_{s} \cdot \operatorname{trace}\left(\mathbf{T T}^{*}\right)=P_{T}\end{cases}
$$

The statistical expectation $E_{\mathbf{s}, \mathbf{n}}\{\}$ is carried out over the data symbols $\mathbf{s}$ and noise samples $\mathbf{n}$. We assume uncorrelated data symbols of average symbol energy $E_{s}$ and zero-mean temporally and spatially white complex Gaussian noise samples with covariance matrix $\sigma_{n}^{2} \mathbf{I}_{M_{R}}$. The trace constraint states that the average total transmit power per $p$-dimensional spatial symbol $\mathbf{s}$, after pre-filtering with $\mathbf{T}$, equals $P_{T}$. The optimization problem stated in (2) is solved using the Lagrange multiplier technique which formulates the constrained cost-function as follows:

$$
\begin{aligned}
C=\operatorname{Min}_{\mathbf{R}, \mathbf{T}} E_{\mathbf{s}, \mathbf{n}}\{ & \left.\|\mathbf{s}-(\mathbf{R H T} \mathbf{s}+\mathbf{R n})\|_{2}^{2}\right\} \\
& +\lambda\left(E_{s} \cdot \operatorname{trace}\left(\mathbf{T} \mathbf{T}^{*}\right)-P_{T}\right)
\end{aligned}
$$


where $\lambda$ is the Lagrange multiplier to be calculated to satisfy the transmit power constraint. Let us introduce the thin SVD [15, p. 72] of the MIMO channel matrix $\mathbf{H}$

$$
\mathbf{H}=\left(\begin{array}{ll}
\mathbf{U}_{p} & \mathbf{U}_{\bar{p}}
\end{array}\right)\left(\begin{array}{cc}
\Sigma_{p} & \mathbf{0} \\
\mathbf{0} & \Sigma_{\bar{p}}
\end{array}\right)\left(\begin{array}{ll}
\mathbf{V}_{p} & \mathbf{V}_{\bar{p}}
\end{array}\right)^{H}
$$

where $\mathbf{U}_{p}$ and $\mathbf{V}_{p}$ are respectively the $M_{R} \times p$ and $M_{T} \times p$ left and right singular vectors associated to the $p$ strongest singular values or spatial subchannels or modes ${ }^{2}$ of $\mathbf{H}$, stacked in decreasing order in the $p \times p$ diagonal matrix $\Sigma_{p}$. $\mathbf{U}_{\bar{p}}$ and $\mathbf{V}_{\bar{p}}$ are the left and right singular vectors associated to the remaining $\left(\operatorname{Min}\left(M_{R}, M_{T}\right)-p\right)$ spatial modes of $\mathbf{H}$, similarly stacked in decreasing order in $\Sigma_{\bar{p}}$. The optimal filter-pair $\{\mathbf{T}, \mathbf{R}\}$ was shown to be [6]

$$
\left\{\begin{array}{l}
\mathbf{T}=\mathbf{V}_{p} \cdot \Sigma_{T} \\
\mathbf{R}=\Sigma_{R} \cdot\left(\mathbf{U}_{p}\right)^{*}
\end{array}\right.
$$

where $\Sigma_{T}$ is the $p \times p$ diagonal power allocation matrix that determines the power distribution among the $p$ spatial modes and is given by

$$
\left\{\begin{array}{l}
\Sigma_{T}^{2}=\left[\frac{\sigma_{n}}{\sqrt{E_{s} \lambda}} \Sigma_{p}^{-1}-\frac{\sigma_{n}^{2}}{E_{s}} \Sigma_{p}^{-2}\right]^{+} \\
\text {subject to: } \operatorname{trace}\left(\Sigma_{T}^{2}\right)=\frac{P_{T}}{E_{s}}
\end{array}\right.
$$

and $\Sigma_{R}$ is the $p \times p$ diagonal complementary equalization matrix given by

$$
\Sigma_{R}=\frac{\sqrt{E_{s} \lambda}}{\sigma_{n}} \Sigma_{T}
$$

The filter-pair MMSE solution $\{\mathbf{T}, \mathbf{R}\}$ of (5) decouples the MIMO channel matrix $\mathbf{H}$ into $p$ parallel spatial subchannels. Furthermore, the analysis of the MMSE power allocation matrix $\Sigma_{T}$ in (6) shows that:

- The MMSE strategy first performs a selection among the $p$ available modes as indicated by $]^{+}$in (6). This boils down to defining a minimum SNR for a mode to be used as described in the following equation:

$$
\left[\begin{array}{c}
\frac{E_{s} \sigma_{1}^{2}}{\sigma_{n}^{2}} \\
\vdots \\
\frac{E_{s} \sigma_{p}^{2}}{\sigma_{n}^{2}}
\end{array}\right] \geq\left[\begin{array}{c}
\lambda \\
\vdots \\
\lambda
\end{array}\right] .
$$

The minimum SNR which appears to be the Lagrange multiplier $\lambda$ is defined by the average total transmit power available $P_{T}$, the receive noise power $\sigma_{n}^{2}$ and the gains of the selected subchannels. The modes that fail to satisfy the criterion of (8) are allocated no power and the power budget is distributed among the selected modes stacked in $\Sigma_{\text {select }}$. It is certainly interesting to point out that the SNR threshold $\lambda$ decreases as the SNR increases which means that eventually all needed $p$ spatial subchannels are used.

- Among the selected modes of $\Sigma_{\text {select }}$, more power is allocated to the weaker modes and vice versa. This is the

\footnotetext{
${ }^{2}$ We alternatively use spatial subchannels and spatial modes to refer to the singular values of $\mathbf{H}$, as these singular values represent the parallel independent spatial subchannels or modes underlying the flat-fading MIMO channel modeled by $\mathbf{H}$.
}

optimal power allocation policy when the same symbol constellation is used over all spatial streams, as earlier postulated and used throughout this paper. Moreover, the MMSE allocation policy leads to an asymptotic zero-forcing behavior as subsequently shown:

$$
\begin{aligned}
\mathbf{R H T}=\left[\frac{\sigma_{n}}{\sqrt{E_{s} \lambda}} \Sigma_{p}^{-1}-\right. & \left.\frac{\sigma_{n}^{2}}{E_{s}} \Sigma_{p}^{-2}\right] \\
& \cdot \frac{\sqrt{E_{s} \lambda}}{\sigma_{n}} \Sigma_{p} \rightarrow \mathbf{I}_{p} \text { when } \sigma_{n} \rightarrow 0 .
\end{aligned}
$$

The discussed conventional joint Tx/Rx MMSE design is derived for a given number of streams $p$ which is initially and arbitrarily chosen and fixed. Hence, accuracy would require us to denote the filter-pair solution as $\left\{\mathbf{T}_{p}, \mathbf{R}_{p}\right\}$. The $p$ spatial multiplexing streams will always be transmitted independently and regardless of the power allocation policy that may, as previously explained, allocate no power to certain modes. The streams assigned to the latter modes are then irremediably lost contributing to a bad overall BER performance. Furthermore, as the SNR increases, these initially disregarded modes will eventually be selected and will monopolize most of the power budget leading to an inefficient power allocation solution. Both previous remarks highlight the influence of the choice of $p$ on the system's performance and power allocation efficiency. Hence, we alternatively propose to include $p$ as a design parameter to be optimized according to the available channel knowledge, for an improved system BER performance.

\section{Mode Selection For the JoInt TRAnsmit AND RECEIVE MMSE DESIGN}

For a fixed number of streams $p$ and a fixed single symbol constellation across these streams, the optimal joint $\mathrm{Tx} / \mathrm{Rx}$ MMSE solution, given by the filter-pair $\left\{\mathbf{T}_{p}, \mathbf{R}_{p}\right\}$ of (5), gives rise to the following minimum mean squared error $\mathrm{MMSE}_{p}$ :

$$
\begin{aligned}
& \operatorname{MMSE}_{p} \\
& =\operatorname{trace}[\underbrace{E_{s}\left(\mathbf{I}_{p}-\Sigma_{T} \Sigma_{p} \Sigma_{R}\right)^{2}}_{\text {imperfect subchannel gain equalization }}+\underbrace{\sigma_{n}^{2} \Sigma_{R}^{2}}_{\text {noise }}]
\end{aligned}
$$

which consists of two distinct contributions namely the imperfect subchannel gain equalization contribution and the noise contribution.

The goal of this work is to identify the optimal number of spatial streams $p_{\text {opt }}$ to be used for transmission, under a fixed rate $R$ constraint. The optimality criterion pertains to the global minimization of the sum mean squared error $\left(\mathrm{MMSE}_{p}\right)_{p} \cdot{ }^{3} \mathrm{We}$ still assume the same symbol constellation across the $p$ used spatial streams for a low-complexity joint Tx/Rx MMSE design. The symbol constellation size $M_{p}$, however, has to be adapted to $p$ in order to satisfy the fixed rate $R$. Moreover, for a meaningful optimization, it is assumed that all the possible constellations have the same minimum distance. Consequently,

\footnotetext{
${ }^{3}$ This criterion does not guarantee the lowest possible BER, which would require a criterion based on the closed-form expression of the system BER. The proposed MMSE-based criterion is simpler and achieves a very good performance.
} 
the average constellation energy $E_{s}$ is variable and depends on the constellation size. Now, the proposed mode-selection criterion can be drawn:

$$
\begin{cases}\operatorname{Min}_{p} & \operatorname{MMSE}_{p} \\ \text { subject to }: & p \times \log _{2}\left(M_{p}\right)=R\end{cases}
$$

For the considered square QAM constellations, the average constellation energy is given by $E_{s}=2\left(M_{p}-1\right) / 3$. Moreover, the rate constraint formulated in (11) defines the constellation size corresponding to a given $p$ as $M_{p}=2^{R / p}$. Consequently, instantiating the $\mathrm{MMSE}_{p}$ general expression of (10) for the considered square QAM constellations, the mode-selection criterion, initially stated in (11), can be refined into

$$
\begin{aligned}
p_{\mathrm{opt}}=\arg \operatorname{Min}_{p}\left\{\operatorname { t r a c e } \left(\frac{2}{3}\left(2^{R / p}-1\right)\right.\right. \\
\left.\left.\times\left(\mathbf{I}_{p}-\Sigma_{T} \Sigma_{p} \Sigma_{R}\right)^{2}+\sigma_{n}^{2} \Sigma_{R}^{2}\right)\right\} .
\end{aligned}
$$

\section{AnAlysis of the Proposed Mode SElection}

In this section, we first propose an intuitive explanation for the existence of the optimal number of streams $p_{\text {opt }}$, which corresponds to the global minimum of the sum mean squared error $\left(\mathrm{MMSE}_{p}\right)_{p}$. Then, we corroborate this explanation with simulation results. Finally, we propose a simplified way to determine the optimal number of streams $p_{\text {opt }}$.

\section{A. Existence of the Optimal Number of Streams $p_{\mathrm{opt}}$}

To get some insight, we analyze the evolution of the minimum mean squared error $\left(\mathrm{MMSE}_{p}\right)_{1 \leq p \leq \operatorname{Min}\left(M_{R}, M_{T}\right)}$ corresponding to the all possible spatial multiplexing scenarios for the previously introduced joint Tx/Rx MMSE design, under the constraints of a fixed average total transmit power $P_{T}$ and a fixed rate $R$. We also analyze the relative evolution of both components of $\mathrm{MMSE}_{p}$ reminded below, namely the contribution of imperfect subchannel equalization and that of the receiver noise:

$\operatorname{MMSE}_{p}=\operatorname{trace}(\underbrace{\frac{2}{3}\left(M_{p}-1\right)\left(\mathbf{I}_{p}-\Sigma_{T} \Sigma_{p} \Sigma_{R}\right)^{2}}_{\text {imperfect subchannel equalization }}+\underbrace{\sigma_{n}^{2} \Sigma_{R}^{2}}_{\text {noise }})$.

We start from the extreme case of $p=1$ stream to convey data at rate $R$. In this case, the total available transmit power is allocated to the strongest mode of gain $\sigma_{1}$, such that $\sigma_{T}^{2}=P_{T} / E_{s}{ }^{4}$ Clearly, this design achieves the maximum receive symbol SNR, as highlighted in [13]. For a large rate $R$, however, the necessary constellation size $M_{1}$ to convey the required rate over a single mode is large. Since, the average total transmit power is constrained, the actual received minimum distance between constellation points is small and thus the induced error rate is large. To be able to convey such high rate while using reasonable constellation sizes, the number of used spatial streams should be increased. For $p$ transmitted data streams using a single common

${ }^{4}$ Where $\Sigma_{T}$ has reduced to the scalar $\sigma_{T}$ in the case of a single stream. constellation, the MMSE power allocation matrix $\Sigma_{T}$ will distribute the available transmit power among the $p$ strongest subchannels in an attempt to balance the SNRs on these subchannels and $\Sigma_{R}$ will equalize the resulting equivalent subchannels. When the number of transmitted streams $p$ is small, the used $p$ strongest subchannels form a balanced subset in terms of gain and diversity, as shown in [12]. Consequently, $\Sigma_{T}$ can perform an efficient power allocation that achieves high SNRs on these subchannels and requires a limited equalization effort. The latter result would translate into a low noise enhancement and hence a low noise contribution to the $\mathrm{MMSE}_{p}$. Nevertheless, the constellation size $M_{p}$ may still be large leading to a large imperfect equalization contribution to the $\mathrm{MMSE}_{p}$ as can be seen in (13). To further scale down the constellation size, the number of transmitted data streams $p$ can be further increased. However, this may lead to the use of weak modes that exhibit lower diversity orders and catastrophic gains [12]. As aforementioned, these weak modes will monopolize most of the available transmit power, penalizing the strongest modes and leading to a severe global reduction in the receive SNR levels. This will eventually translate into a larger equalization matrix $\Sigma_{R}$ and noise contribution to $\mathrm{MMSE}_{p}$.

The previous analysis shows that, for a fixed rate $R$, there exists an optimal number of streams $p_{\text {opt }}$ that enables a reasonable constellation size $M_{\text {opt }}$ while achieving the most efficient power distribution that balances the achieved SNRs on the used subchannels and the receive noise enhancement.

\section{B. Analysis of the Dependence of $p_{\mathrm{opt}}$ on Various System Parameters}

For all simulations, we consider the case of a $(6,6)$ MIMO set-up, where both transmitter and receiver are assumed to have perfect CSI. The MIMO channel is stationary Rayleigh flat-fading, modeled by an $M_{R} \times M_{T}$ matrix with i.i.d unitvariance zero-mean complex Gaussian entries. Moreover, the average total transmit power is fixed to $P_{T}=1$. In all the following, SNR stands for the average total transmit power over the noise power.

Fig. 2 illustrates, for a typical $(6,6)$ MIMO channel, the existence of the optimal number of spatial streams, $p_{\text {opt }}$, that globally minimizes the system's sum mean squared error $\left(\mathrm{MMSE}_{p}\right)_{p}$ according to (12). Fig. 2 is plotted for an SNR of $20 \mathrm{~dB}$ and a spectral efficiency $R$ equal to $12 \mathrm{bits} / \mathrm{s} / \mathrm{Hz}$. This rate corresponds to a full spatial multiplexing strategy $(p=6)$ using QPSK symbol modulation. Fig. 3 further plots the distribution of the previous optimal number of streams, $p_{\text {opt }}$, evaluated over 10000 channel realizations. Interestingly, it shows that there exists a dominant $p_{\text {opt }}$ value for the given system parameters and channel statistics, in this case $p_{\text {opt }}=3$. This is due to the fact that, as proved in [12] for i.i.d complex Gaussian MIMO channels, the spatial modes have strictly decreasing gains and diversity orders until the last subchannel that exhibits a catastrophic less than first-order diversity. Consequently, it is predictable that there will be a dominant $p_{\text {opt }}$ value. The importance of the marginal values, however, will depend on the granularity of the available constellation sizes and the diversity orders of the weaker used modes. These modes, in fact, exhibit a larger variability and could alternatively be retained or discarded, depending on the channel realization. The remarkable 


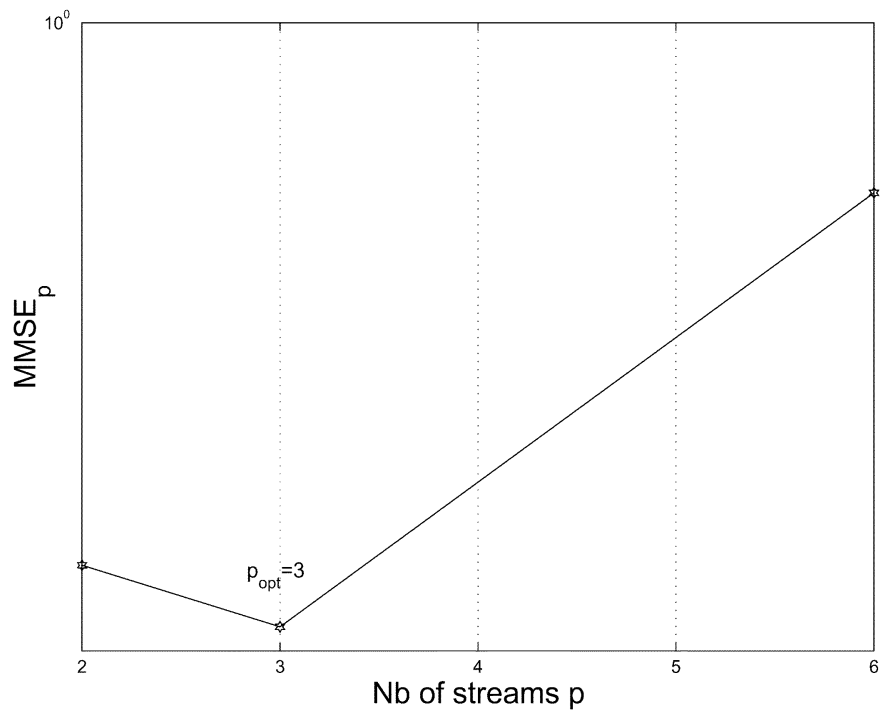

Fig. 2. Illustration of the existence of the optimal number of streams $p_{\text {opt }}$, for a sample $(6,6)$ MIMO channel at SNR $=20 \mathrm{~dB}$ and $R=12 \mathrm{bits} / \mathrm{s} / \mathrm{Hz}$.

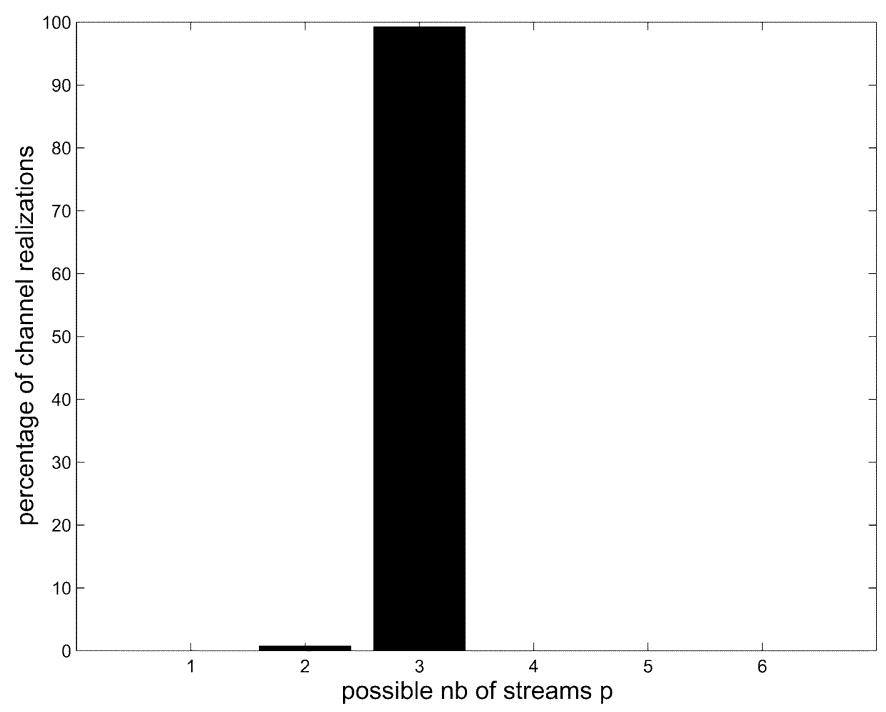

Fig. 3. $p_{\text {opt }}$ 's distribution for a (6,6) MIMO system at $S N R=20 \mathrm{~dB}$ and $R=12$ bits $/ \mathrm{s} / \mathrm{Hz}$.

existence of a dominant $p_{\text {opt }}$ value will be exploited later on to propose an average mode selection, which uses this dominant $p_{\text {opt }}$ value for all channel realizations rather than adapting $p_{\text {opt }}$ for each channel realization. Both proposed instantaneous and average mode selections pertain to rate-constrained MIMO link adaptation techniques [14] to be used in conjunction with the joint Tx/Rx MMSE design for minimum-BER performance. Based on the previous remarks, the performance of the average mode selection is expected to depend on how dominant the chosen $p_{\text {opt }}$ value is or in other words the relative importance of the marginal $p_{\text {opt }}$ values. These effects will be illustrated in Section V.

It is interesting to investigate $p_{\text {opt }}$ 's dependence on the SNR. For a sample channel of the previously considered $(6,6)$ MIMO set-up at $\mathrm{R}=12 \mathrm{bits} / \mathrm{s} / \mathrm{Hz}$, Fig. 4 plots the system's $\mathrm{MMSE}_{p}$ for different SNR values. It reveals that, starting from a given

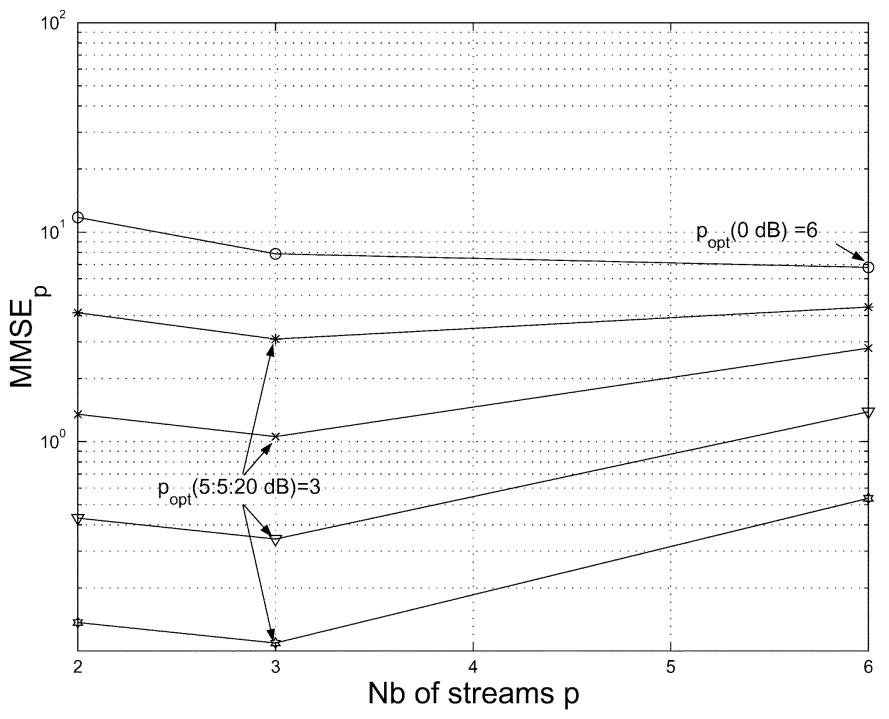

Fig. 4. Illustration of $p_{\text {opt }}$ 's dependence on the SNR, for a sample $(6,6)$ MIMO channel at $R=12 \mathrm{bits} / \mathrm{s} / \mathrm{Hz}$.

SNR value between 0 and $5 \mathrm{~dB}$, the optimal number of streams $p_{\text {opt }}$ remains the same. The fact that, for very low SNR values, the determined $p_{\text {opt }}$ value is different from the common value in most of the SNR range, is due the failure of the MMSE criterion to determine the actual optimal $p_{\text {opt }}$ value at very low SNR. This can be explained by the failure of MMSE criterion to relate to the actual BER performance in the very low SNR range. Indeed, as previously highlighted, for low SNR values, the full spatial multiplexing power allocation will certainly allocate no power to the weakest modes that fail to reach a given minimum gain, as highlighted by (6) and the subsequent discussion. In spite of the irremediably lost data and the resulting large BER figure, the exhibited $\mathrm{MMSE}_{p}$, given by (12), will paradoxically be the smallest, mainly because the corresponding constellation exhibits the smallest average energy $E_{s}$. Consequently, the MMSE criterion is relevant starting from the SNR value, at which all modes are allocated power in the case of full spatial multiplexing. Beyond this SNR value, we can determine the closed-form expression of the Lagrange multiplier $\lambda$ and re-write (6) as follows:

$$
\left\{\begin{array}{l}
\Sigma_{T}^{2}=\frac{\sigma_{n}}{\sqrt{E_{s} \lambda}} \Sigma_{p}^{-1}-\frac{\sigma_{n}^{2}}{E_{s}} \Sigma_{p}^{-2} \\
\text { where: } \quad \lambda=\left(\frac{\sqrt{E_{s}} \sigma_{n} \operatorname{trace}\left(\Sigma_{p}^{-1}\right)}{P_{T}+\sigma_{n}^{2} \operatorname{trace}\left(\Sigma_{p}^{-2}\right)}\right)^{2} .
\end{array}\right.
$$

The equalization matrix is still $\Sigma_{r}=\left(\sqrt{E_{s} \lambda} / \sigma_{n}\right) \Sigma_{t}$, as earlier defined in (7). Using the previous expressions of $\Sigma_{T}, \Sigma_{R}$ and $\lambda$ in the expression of $\mathrm{MMSE}_{p}$ given in (13), we get:

$$
\operatorname{MMSE}_{p}=\frac{\frac{2}{3}\left(M_{p}-1\right) \operatorname{trace}\left(\Sigma_{p}^{-1}\right)^{2}}{\operatorname{SNR}+\operatorname{trace}\left(\Sigma_{p}^{-2}\right)}
$$

where SNR was previously identified as the average total transmit power over the noise power $\left(P_{T} / \sigma_{n}^{2}\right)$. Consequently, the final mode-selection criterion reads:

$$
p_{\text {opt }}=\arg \operatorname{Min}_{p}\left\{\frac{\frac{2}{3}\left(M_{p}-1\right) \operatorname{trace}\left(\Sigma_{p}^{-1}\right)^{2}}{\mathrm{SNR}+\operatorname{trace}\left(\Sigma_{p}^{-2}\right)}\right\} .
$$


On the one hand, the examination of the numerator of the $\mathrm{MMSE}_{p}$ expression in (15) and the evaluation of the its evolution for different values of $p$ show that it is a convex function of $p$. As such, it exhibits a minimum value. On the other hand, the examination of the denominator shows that it is a monotonously increasing function of $p$. Nevertheless, it was observed that, for the relevant SNR range, ${ }^{5}$ the attained values are not sufficient to actually alter neither the convex nature of the numerator nor the position of its minimum $p_{o}$. Furthermore, as the SNR increases, it gradually outweighs trace $\left(\Sigma_{p}^{-2}\right)$ and finally dominates the denominator. Since, SNR is independent of $p$, the previous remark establishes that the evolution of $\mathrm{MMSE}_{p}$ as a function of $p$ is then ${ }^{6}$ exclusively dependent on the numerator. The SNR value, at which these effects are observed, is clearly related to the rank conditioning of the channel. In fact, the smaller the gain of the last mode is, the larger the SNR value that outweighs trace $\left(\Sigma_{p}^{-2}\right)$. Nevertheless, the smaller the gain of the last mode, the larger the value of the numerator for the case of full spatial multiplexing and the more difficult it is to alter the convexity of the numerator. As a conclusion, the denominator was seen not to alter neither the convexity of the numerator nor the number of streams $p_{o}$ corresponding to its minimum. As result, $p_{o}$ corresponding to the minimum of the numerator can be identified as $p_{\text {opt }}$ corresponding to the minimum of $\mathrm{MMSE}_{p}$. Since the denominator is clearly independent of the SNR, we can conclude that, except for very low SNR values, the optimal number of streams $p_{\text {opt }}$ is independent of the SNR. The optimality here pertains to the MMSE minimization.

\section{Simplified Mode Selection Cost-Function}

The previous analysis suggests that it is sufficient to simply consider the numerator of $\mathrm{MMSE}_{p}$ in (16) to determine the optimal number of streams $p_{\text {opt }}$. We subsequently refer to the numerator of $\mathrm{MMSE}_{p}$ as a simplified error $\operatorname{Err}_{p}$ :

$$
\operatorname{Err}_{p}=\frac{2}{3}\left(2^{R / p}-1\right) \cdot \operatorname{trace}\left(\Sigma_{p}^{-1}\right)^{2} .
$$

We managed to reduce the complex $\mathrm{MMSE}_{p}$ expression of (13), which depends on a large amount of parameters and which is composed of inter-dependent quantities, into a simplified expression $\operatorname{Err}_{p}$ that preserves the same minimum and thus the same $p_{\text {opt }}$ across all the SNR range, except very low SNRs as explained in the previous subsection. This is confirmed by Fig. 5, for the previously introduced $(6,6)$ MIMO set-up at $R=$ $12 \mathrm{bits} / \mathrm{s} / \mathrm{Hz}$. The simplified $\operatorname{Err}_{p}$, expressed in (17), is simply the product of two terms, each depending on a single system parameter namely the channel singular matrix $\Sigma_{p}$ and the reference rate $R$. Consequently, the proposed simplified $\operatorname{Err}_{p}$ fairly eases $p_{\text {opt }}$ 's computation and more importantly does not require receiver noise power estimation. Finally, the simplified mode-selection criterion can be drawn:

$$
p_{\text {opt }} \approx \arg \operatorname{Min}_{p}\left\{\frac{2}{3}\left(2^{R / p}-1\right) \cdot \operatorname{trace}\left(\Sigma_{p}^{-1}\right)^{2}\right\} .
$$

In the sequel, Monte-Carlo BER analysis will show that this simplified mode-selection criterion succeeds, unlike the mode-

\footnotetext{
${ }^{5}$ For which all modes are allocated power.

${ }^{6}$ When SNR dominates the value of the denominator.
}

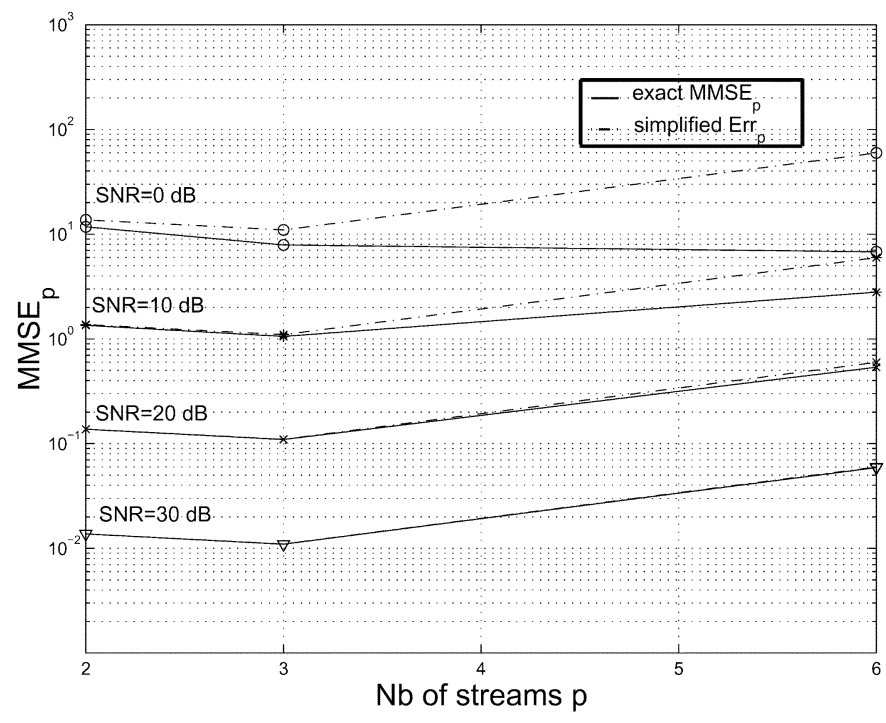

Fig. 5. Comparison of the simplified $\operatorname{Err}_{p}$ and the exact $\mathrm{MMSE}_{p}$, for a sample $(6,6)$ MIMO channel at various SNR values.

selection criterion based on the exact $\mathrm{MMSE}_{p}$ expression, in determining the optimal number of streams $p_{\text {opt }}$ that minimizes the BER at very low SNR values. This further suggests that the actual optimal number of streams $p_{\text {opt }}$ is independent of the SNR.

\section{PERformance AnALYSiS}

In the previous section, we have proposed a mode-selection approach that determines the optimal number of spatial streams to be used by the joint Tx/Rx MMSE design, for each channel realization. The optimality criterion pertains to the global minimization of the sum mean squared error. We have also highlighted the existence of a dominant $p_{\text {opt }}$ value for given system parameters and the considered i.i.d complex Gaussian MIMO channel model. Based on that, we have proposed an average mode selection that chooses the latter dominant value $p_{\text {opt }}$ for all channel realizations, instead of adapting $p_{\text {opt }}$ to each channel realization. In this section, we first investigate the BER performance improvement provided by both the "per-channel" and the average mode selection approaches over the conventional joint Tx/Rx MMSE design, which chooses arbitrarily the number of spatial streams to be used. Then, we compare the performance of our mode selection to that of a practical adaptive loading strategy [11]. The latter not only adapts the number of spatial streams $p$ but also assumes the additional flexibility of variable constellation sizes across these spatial streams.

\section{A. Mode Selection Versus the Conventional Joint Tx/Rx MMSE}

Fig. 6 depicts the performance of the conventional joint Tx/Rx MMSE design with various fixed number of streams $p$ as well as with both our exact and simplified mode selection, for the previously introduced $(6,6)$ MIMO system at $R=12 \mathrm{bits} / \mathrm{s} / \mathrm{Hz}$. It clearly corroborates the fact that the simplified mode selection based on (18), unlike the exact mode selection based on (12), succeeds in identifying the optimal number of streams $p_{\text {opt }}$ at very low SNR values. For the remaining SNR range, both mode selection criteria exhibit the 


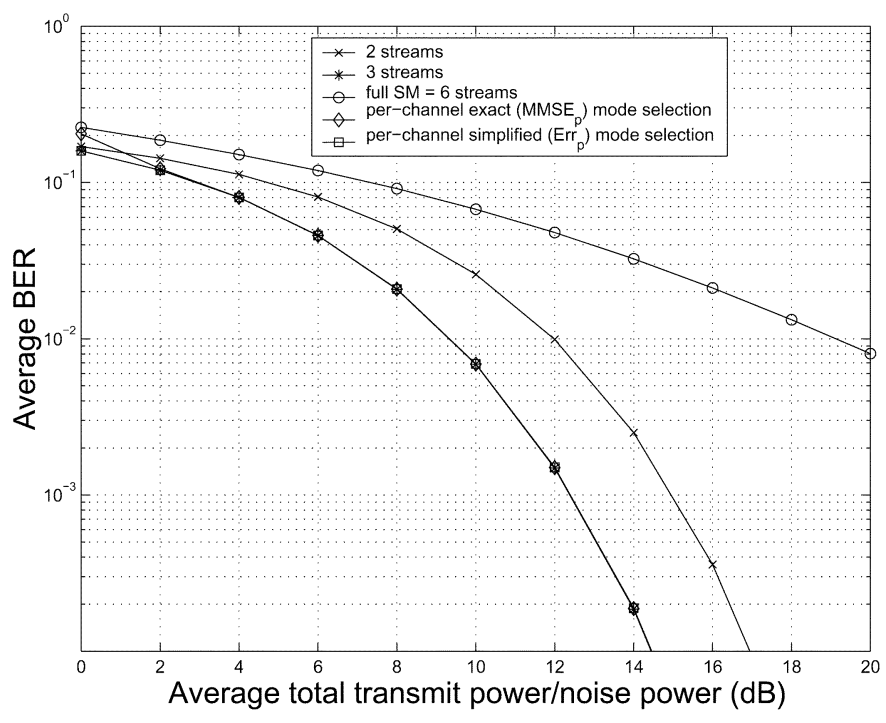

Fig. 6. Joint Tx/Rx MMSE design BER performance with different number of streams, for a $(6,6)$ MIMO system at $R=12 \mathrm{bits} / \mathrm{s} / \mathrm{Hz}$.

same performance. In all the following, we retain only the simplified mode selection and simply refer to it as mode selection. Fig. 6, more importantly, shows that our mode selection enables the best joint Tx/Rx MMSE design BER performance, outperforming those exhibited when full spatial multiplexing is used or when an arbitrary number of streams is chosen $(p=2)$. Indeed, our mode selection offers, respectively, $10.4 \mathrm{~dB}$ and $2.7 \mathrm{~dB}$ SNR gain over the full spatial multiplexing and the $(p=2)$ scenarios, at $\mathrm{BER}=10^{-2}$. In fact, as illustrated by Fig. 3, our "per-channel" mode selection almost always chooses $p_{\mathrm{opt}}=3$. This explains why its performance is basically the same as that of the "average" mode selection that chooses $p=3$ for all channel realizations. Nevertheless, this result only holds for the considered constellation set, namely \{QPSK, 16QAM, 64QAM\}. As earlier highlighted, allowing more granularity in the constellation sizes will certainly reduce the dominance of a single $p_{\text {opt }}$ value and enhance the importance of other marginal $p_{\text {opt }}$ values. This will lead to a performance gap between the per-channel and the average mode selection. Through restricting transmission to the $p_{\mathrm{opt}}=3$ strongest modes, for spectral efficiency $R=12 \mathrm{bits} / \mathrm{s} / \mathrm{Hz}$, both mode selection strategies wisely avoid the remaining weaker modes that exhibit lower diversity orders, as argued in [12]. The same weak modes that dominate the performance of the full spatial multiplexing joint Tx/Rx MMSE design in Fig. 6. Indeed, it is because mode selection chooses to transmit only on the $p_{\text {opt }}$ higher-diversity modes that it better exploits the available MIMO spatial diversity, as illustrated by its higher BER curve slope. Nevertheless, the decisive advantage of $p_{\mathrm{opt}}=3$ over the $(p=2)$ scenario, which even better exploits the system spatial diversity, is that it allows a more robust constellation, namely 16QAM instead of the 64QAM required when $p=2$. In this particular case, the former constellation relatively achieves lower BER figures given the joint Tx/Rx MMSE design's transmit power allocation and the statistics of the MIMO channel modes. In a nutshell, as highlighted in Section IV-A, mode selection enables the best performance because it succeeds to determine the optimal pair

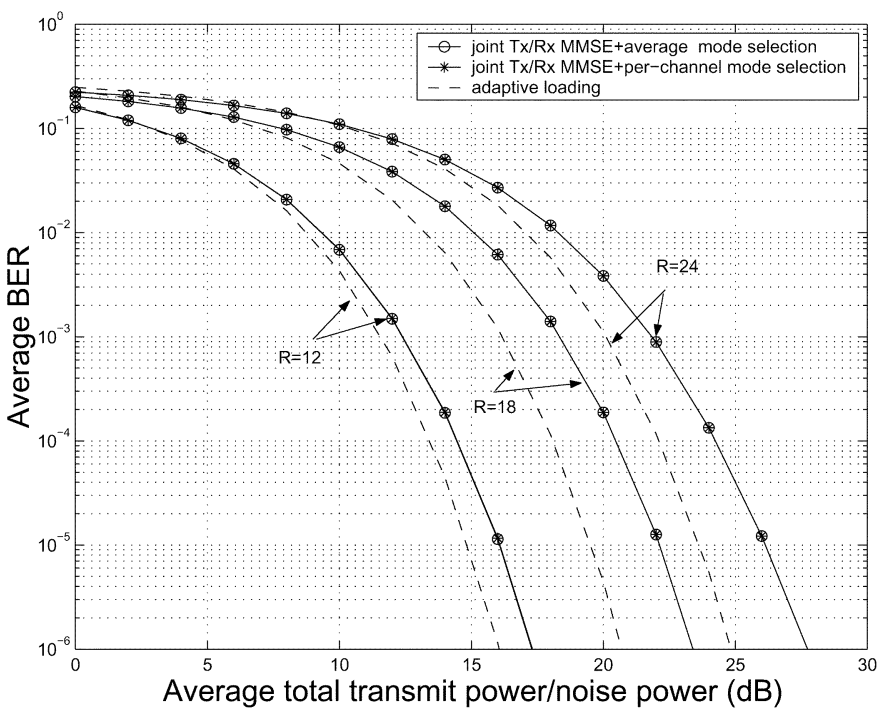

Fig. 7. Comparison of the joint Tx/Rx MMSE with mode selection to adaptive loading at different rates $R$, for a $(6,6)$ MIMO system.

$\left(p_{\text {opt }}, M_{\text {opt }}\right)$ that strikes the best compromise between the spatial diversity exploitation and constellation size.

\section{B. Mode Selection Versus Adaptive Loading}

The adaptive loading strategy, herein considered, is simply the practical Fischer's adaptive loading algorithm [11]. This algorithm was initially proposed for multicarrier systems. Nevertheless, it directly applies for a MIMO system, where an SVD is used to decouple the MIMO channel into its parallel spatial eigen-subchannels or modes, which are completely analogous to the orthogonal carriers of a multicarrier system. Hence, the considered adaptive loading design first performs the SVD of MIMO channel to access the parallel spatial modes. Fischer's adaptive loading algorithm [11] is then used to determine, using the knowledge of the current channel realization, the optimal assignment of the $R$ bits on the decoupled spatial modes, such that equal minimum symbol-error rate (SER) is achieved on the used modes. Consequently, strong spatial modes are loaded with large constellation sizes whereas weak modes carry small constellations or are dropped if their gains are below a given threshold. This scheme has excellent performance, as illustrated in Fig. 7 for the considered $(6,6)$ MIMO system at spectral efficiencies $R=\{12,18,24\}$ bits/s/Hz. Furthermore, adaptive loading is shown to outperform the joint Tx/Rx MMSE with our mode selection. For instance, adaptive loading appears to offer the largest SNR gain over the optimized joint Tx/Rx MMSE design at spectral efficiency $R=18 \mathrm{bits} / \mathrm{s} / \mathrm{Hz}$. In this case, a $2.1 \mathrm{~dB}$ SNR gain is observed at BER $=10^{-3}$. It is worthwhile to point out that the bit and power allocation of Fischer's adaptive loading algorithm, as can be verified in [11], does not depend on the SNR. We have also highlighted that the optimal number of streams, determined by our mode selection, is also independent of the SNR. Consequently, both schemes keep the same bit assignment across the whole SNR range, for a given spectral efficiency $R$. We believe that the system parameters that determine these initial assignments, namely the MIMO system dimensions, the MIMO channel statistics, the desired spectral efficiency $R$ and the granularity of the available constellation 
family, are the parameters that determine the BER performance difference between our mode selection and adaptive loading. Clearly, the better adaptive loading performance is due to its additional flexibility of assigning different constellation sizes to different modes, whereas the joint Tx/Rx MMSE design assumes a single constellation across these modes. This higher flexibility, however, comes at the cost of a higher complexity to determine the bit assignments and signaling overhead to support different constellation sizes across the modes. These complexity and signaling overhead requirements clearly outgrow those of the "per-channel" mode selection and a fortiori those of the average mode selection. The latter scheme is further confirmed to exhibit the BER performance of the "per-channel" mode selection without a per-channel adaptation, for the herein considered i.i.d complex Gaussian MIMO channel model. Consequently, it is our preferred scheme as it closely approaches the optimal performance of adaptive loading while exhibiting a much lower complexity and signaling overhead.

\section{CONCLUSIONS}

In this paper, we proposed a novel selection-diversity technique, so-called mode selection, that optimally selects, based on the knowledge of the current channel realization, the number of spatial streams used by the spatial multiplexing joint $T x / R x$ MMSE design in order to optimize the BER performance. The optimality criterion pertains to the global minimization of the system's sum mean squared error. We have assessed the significant improvement in terms of BER performance, our mode selection provides over the conventional joint $\mathrm{Tx} / \mathrm{Rx}$ MMSE design. Such significant improvement were shown to be due to the more efficient transmit power allocation and the better exploitation of the available spatial diversity enabled by the proposed mode selection. Furthermore, when our mode selection is applied, the joint Tx/Rx MMSE design was shown to closely approach the optimal performance of adaptive loading while exhibiting lower complexity and signaling overhead requirements.

\section{REFERENCES}

[1] G. J. Foschini and M. J. Gans, "On limits of wireless communications in a fading environment when using multiple antennas," Wireless Personal Commun., vol. 6, pp. 311-335, Mar. 1998.

[2] A. J. Paulraj and T. Kailath, "Increasing Capacity in Wireless Broadcast Systems Using Distributed Transmission/Directional Reception," U.S. Patent 5,345,599, Sep. 6, 1994

[3] G. J. Foschini, "Layered space-time architecture for wireless communication in a fading environment when using multi-element antennas," Bell Labs Tech. J., vol. 1, no. 2, pp. 41-59, 1996.

[4] A. Medles and D. T. M. Slock, "Full diversity spatial multiplexing based on SISO channel coding, spatial spreading and delay diversity," in Proc. IEEE Benelux Signal Processing Symp., Leuven, Belgium, Mar. 2002, pp. 149-152.

[5] J. Yang and S. Roy, "On joint transmitter and receiver optimization for multiple-input multiple-output (MIMO) transmission systems," IEEE Trans. Commun., vol. 42, no. 12, Dec. 1994.

[6] H. Sampath and A. J. Paulraj, "Joint transmit and receive optimization for high data rate wireless communication using multiple antennas," in Proc. Asilomar Conf. Signals, Systems and Computers, Asilomar, CA, Nov. 1999, pp. 215-219.

[7] H. Sampath, P. Stoica, and A. J. Paulraj, "Generalized linear precoder and decoder design for MIMO channels using the weighted mmse criterion," IEEE Trans. Commun., vol. 49, no. 12, pp. 2198-2206, Dec. 2001.

[8] A. Scaglione, P. Stoica, S. Barbarossa, G. B. Giannakis, and H. Sampath, "Optimal designs for space-time linear precoders and decoders," IEEE Trans. Signal Processing, vol. 50, no. 5, pp. 1051-1064, May 2002.
[9] G. G. Raleigh and J. M. Cioffi, "Spatio-temporal coding for wireless communications," IEEE Trans. Commun., vol. 46, no. 3, pp. 357-366, Mar. 1998.

[10] D. Hughes-Hartogs, "Ensemble Modem Structure for Imperfect Transmission Media," U.S Patent 4,833,706, May 23, 1989.

[11] R. F. H. Fischer and J. B. Huber, "A new loading algorithm for discrete multitone transmission," in Proc. IEEE GLOBECOM, London, U.K., Nov. 1996, pp. 724-728.

[12] J. B. Andersen, "Array gain and capacity for known random channels with multiple elements arrays at both ends," IEEE J. Select. Areas Commun., vol. 18, no. 11, pp. 2172-2178, Nov. 2000.

[13] A. J. Paulraj and C. B. Papadias, "Space-time processing for wireless communications," IEEE Signal Process. Mag., vol. 14, no. 6, pp. 49-83, Nov. 1997.

[14] S. Catreux, V. Erceg, D. Gesbert, and R. W. Heath Jr, "Adaptive modulation and MIMO coding for broadband wireless data networks," IEEE Commun. Mag., vol. 40, no. 6, pp. 108-115, Jun. 2002.

[15] G. H. Golub and C. F. Van Loan, Matrix Computations. Baltimore, MD: John Hopkins Univ. Press, 1996.

[16] J. G. Proakis, Digital Communications, 3rd ed. New York: McGrawHill, 1995

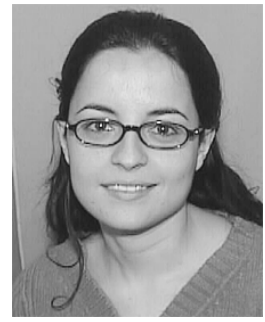

Nadia Khaled (S'02) was born in Rabat, Morocco, in 1977. She received the M.Sc. degree in electrical engineering from l'Ecole Nationale Supérieure d'Electrotechnique, d'Electronique, d'Informatique, d'Hydraulique et des Télécommunications (ENSEEIHT), Toulouse, France, in 2000. Since May 2001, she has been pursuing the Ph.D. degree with the wireless research group of IMEC, Leuven, Belgium, at the Katholieke Universiteit Leuven.

Her research interests lie in the area of signal processing for wireless communications, particularly MIMO techniques and transmit optimization schemes.

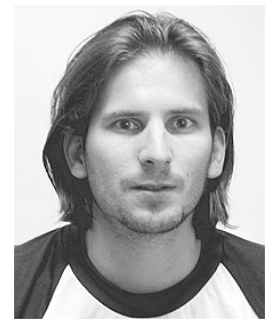

Steven Thoen was born in Leuven, Belgium, in 1974. He received the M.Sc. degree in electrical engineering and the Ph.D. degree in communications engineering from the Katholieke Universiteit Leuven, Belgium, in 1997 and in 2002, respectively.

From October 1997 until May 2002, he was with the wireless systems (WISE) group of IMEC, Leuven, Belgium as a Ph.D. student at the Katholieke Universiteit Leuven, supported by an FWO scholarship. From October 1998 to November 1998, he was a visiting researcher at the Information Systems Laboratory of Stanford University, Palo Alto, CA. In July 2002, he joined Resonext Communications where he worked on the design and implementation of advanced WLAN modems. Currently, he is working as a staff system engineer in the WLAN group of RF Micro Devices. His research interests include systems design, signal processing and digital communications systems, with particular regard to transmit optimization and MIMO transmission.

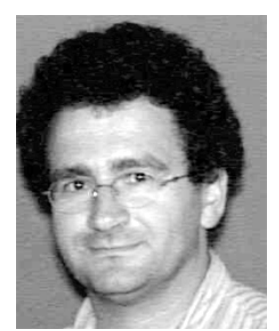

Luc Deneire (M'99) received the Engineering degree in electronics from the University of Liège, Belgium, in 1988, the Engineering degree in telecommunications from the University of Louvain-La-Neuve, Belgium, in 1994, and the Ph.D. degree in signal processing from Eurécom, Sophia-Antipolis, France, in 1998. During this time, he was a Marie Curie Fellow grant holder.

From 1999 to 2002, he was a senior researcher at IMEC, the largest European independent research institute in Micro-Electronics and then joined the University of Nice, France, as an Associate Professor. He is working on the signal processing algorithms involved in wireless communications, specifically for third-generation mobile networks, wireless LANs and wireless personal area networks. His main interests are blind and semi-blind equalization and channe estimation, modulation theory, multiple access schemes, smart antennas, and link adaptation. 